\title{
Prevalence and factors associated with fertility desire among people living with HIV: A systematic review and meta-analysis
}

\author{
Xiang Yan ${ }^{1 \oplus}$, Jie Du ${ }^{2 \odot}$, GuoPing Ji $\oplus^{1,3 *}$ \\ 1 Department of Epidemiology and Health Statistics, School of Public Health, Anhui Medical University, \\ Hefei, Anhui, China, 2 The First Affiliated Hospital of USTC, Hefei, Anhui, China, 3 Anhui Provincial Center \\ for Women's and Children's Health, Hefei, Anhui, China \\ - These authors contributed equally to this work. \\ *429794069@qq.com
}

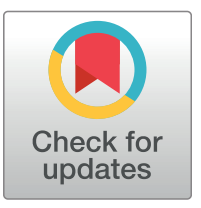

\section{openaccess}

Citation: Yan X, Du J, Ji G (2021) Prevalence and factors associated with fertility desire among people living with HIV: A systematic review and meta-analysis. PLOS ONE 16(3): e0248872. https:// doi.org/10.1371/journal.pone.0248872

Editor: Joseph K.B. Matovu, Makerere University School of Public Health, UGANDA

Received: June 27, 2020

Accepted: March 7, 2021

Published: March 18, 2021

Copyright: @ 2021 Yan et al. This is an open access article distributed under the terms of the Creative Commons Attribution License, which permits unrestricted use, distribution, and reproduction in any medium, provided the original author and source are credited.

Data Availability Statement: All relevant data are within the paper and its Supporting Information files.

Funding: The author(s) received no specific funding for this work.

Competing interests: The authors have declared that no competing interests exist.

\section{Abstract}

\section{Background}

The fertility desire of people living with HIV (PLHIV) has been rising in the past decade. However, there are many studies among which the association remains controversial between the fertility desire of HIV-infected persons and antiretroviral therapy (ART), sex, marital status, and educational level.

\section{Methods}

We performed a literature search of these meta-analyses in PubMed, the Cochrane Library, Web of Science and ScienceDirect in November 2019. We also reviewed references of eligible studies to complement the search. We used pooled odds ratios (ORs) and $95 \%$ confidence intervals (Cls) with a random-effects model and a fixed-effects model to estimate the association between fertility desire among PLHIV and ART, sex, age, marital status, educational level, and number of children. Subgroups with I square values $\left(I^{2}\right)$ and sensitivity analyses were performed to assess the heterogeneity and the stability of the overall ORs, respectively. We evaluated publication bias using Egger's test and a visual inspection of the symmetry in funnel plots.

\section{Results}

In these meta-analyses 50 articles were included with 22,367 subjects. The pooled prevalence of fertility desire among PLHIV was estimated to be $42.04 \%$. The pooled analyses showed that the fertility desire of PLHIV is associated with ART (OR $=1.11,95 \% \mathrm{Cl}: 1.00-$ 1.23, $\mathrm{P}=0.043)$, sex $(\mathrm{OR}=1.51,95 \% \mathrm{Cl}: 1.10-2.09)$, age $(\mathrm{OR}=2.65,95 \% \mathrm{Cl}: 2.24-3.14)$, marital status (OR $=1.34,95 \% \mathrm{Cl}: 1.08-1.66)$, educational level $(\mathrm{OR}=0.85,95 \% \mathrm{Cl}: 0.73-$ $1.00, P=0.047)$ and the number of children $(\mathrm{OR}=3.99,95 \% \mathrm{Cl}: 3.06-5.20)$. PLHIV who are on ART, are male, are younger than 30 , are married/cohabiting, have received a secondary education or above, and are childless have a higher prevalence of fertility desire. The two factors of age and the number of children, in particular demonstrated a strong significant 
association with fertility desire. We found moderate heterogeneity in the meta-analyses of age and educational level and high heterogeneity in the meta-analyses of sex, marital status and number of children. Publication bias was detected in the meta-analyses of the association of fertility with sex and educational level.

\section{Conclusion}

This study demonstrates that the prevalence of fertility desire among HIV-infected people is 42.04\%, and the fertility desire among PLHIV is associated with ART experience, sex, age, marital status, the number of children, and educational level. Since a majority of PLHIV are of reproductive age, it is necessary to support PLHIV in terms of their needs regarding reproductive decision-making. Through counseling and reproductive health care, further measures to prevent the horizontal and vertical transmission of HIV should be taken.

\section{Introduction}

According to the 2019 reports of Joint United Nations Programme on HIV/AIDS (UNAIDS), there were approximately 37.9 million people living with HIV (PLHIV) across the world, among whom 1.47 million were of reproductive age [1]. It is common for them to want to get married and start a family. However, the prevalence of fertility desire/intention among PLHIV has been low for the past two decades due to poor health status, fear of infecting one's spouse or fetus, and discouraging policies in many countries [2]. In 2000, a study conducted in Europe showed that the pregnancy rate trended to decrease in women after receiving a diagnosis of HIV while the rate of abortion had increased [3]. Lewis et al showed that HIV-infected women in Sub-Saharan Africa have a lower fertility rate than their non-infected counterparts [4].

With more access to highly active antiretroviral therapy (HAART), nevertheless, the quality of life for PLHIV has significantly improved, and their life span is expected to be longer $[5,6]$. As a result, their fertility desire or intention has risen [7, 8]. A study from Ethiopia indicated that the prevalence of fertility desire among HIV-positive individuals increased from $20.0 \%$ in 2010 to $42.1 \%$ in $2013[9,10]$. A similar growing tendency toward the desire to reproduce has been found in other regions as well [11]. A number of studies have pointed to many factors that could influence the fertility desire of PLHIV. For example, HIV-positive individuals who are young tend to have a higher prevalence of fertility desire $[12,13]$. Decreased fertility desire is related to divorce or separation compared to being married, and to having at least one child in contrast to having no children [12-14]. Several studies have revealed that educational status is a predicator of fertility desire [15]. In addition, HIV disclosure to sexual partners could affect the fertility desire of HIV-infected people $[14,16]$.

Since the fertility desires of PLHIV is tied to sexual practices and pregnancy, it is crucial to prevent horizontal transmission between partners and mother-to-child transmission (MTCT). Previous studies indicate that the viral load of PLHIV on ART could be suppressed; therefore, the risk of transmitting HIV to sexual partners could decrease or even be eliminated [17]. Many measures for sero-discordant couples, such as artificial insemination, timed unprotected intercourse, assisted reproductive techniques, sperm washing and pre-exposure prophylaxis, could be used to reduce HIV horizontal transmission [18-20]. There are also many means of preventing the mother-to-child transmission of HIV (PMTCT), including HIV testing and counseling, the use of ARV drugs, safe delivery, and safe breastfeeding. As a consequence, fertility desire among PLHIV not only has significant implications for such individuals, their 
partners, and fetuses, but also plays a critical role in preventing HIV transmission and providing reproductive health care.

Studies have shown that the fertility desire of PLHIV is associated with many sociodemographic factors, while there are many inconsistent conclusions regarding whether being on ART is related to higher fertility desire [21-23]. A previous meta-analysis conducted in 2013 explore the associations of fertility with some variables including ART experience, sex, age, the number of children, and educational level [24]. In that study, however, the results showed no significant association of fertility desire with ART experience and sex and hence might not estimate the true effect size; it should be noted that the association between fertility desire and the factors stated above remains inconsistent. Since this study involves more literature on research performed from 2013 to 2019, the objective is to offer a broad description of fertility desire among PLHIV, and was to underscore the strength of the association between these factors and fertility desire.

\section{Methods}

We reported this meta-analysis with reference to the Preferred Reporting Items for Systematic Review and Meta-Analyses (PRISMA) guidelines [25] (S1 Table).

\section{Search strategy}

The literature searching was conducted in PubMed, Cochrane Library, Web of Science and ScienceDirect by following the search strategy and completed before November 24, 2019 (S2 Table): (Fertility desire OR Fertility intention OR desire to have children OR Reproductive intention OR Reproductive decision making OR Desire for child OR Childbearing desire OR Childbearing intention OR parenthood OR fatherhood OR motherhood OR maternity OR paternity) AND (HIV OR people living with HIV OR HIV-positive OR HIV-infected). We extracted all relevant studies by reading theirs titles, abstracts, keywords and full texts. The references of the included studies and any relevant meta-analyses were also reviewed.

\section{Inclusion and exclusion criteria}

The inclusion criteria were: 1)the outcome of interest was fertility desire or intention in the future; 2) the female subjects in the study were all of childbearing age and able to achieve pregnancy; 3)the studies reported the prevalence of fertility desire among PLHIV or least one of the selected associated factors (sex, age, marital status, educational level, number of children, and ART experience); 4)the details of the sample/subsample could be extracted; 5)observational studies published from 2000 to November 2019; and 6)quantitative studies. Studies were excluded if 1) the study was a review, a qualitive study or only an abstract; 2) the study's objective was irrelevant; 3 ) the study's subjects were ineligible. and 4) data were not available or adequate.

\section{Data extraction}

The following data from the eligible studies were collected: the first author's name, publication year, the country/location where the study was conducted, the study design, the sample size, the number of men and women, the prevalence of fertility desire among PLHIV, and the study's quality assessment score. If the adjusted odds ratio (OR) values and their $95 \%$ confidence intervals (CIs) were unavailable, we directly extracted or calculated the ORs with corresponding 95\% CIs using the raw data. Variables of age, marital status, the number of children and educational level were respectively dichotomized as aged below 30 years vs aged 30 years and above, currently married/cohabiting vs not currently married (single, widowed, separated or divorced), having children vs having one or more children, and up to primary education vs 
secondary education or above. In this review, two investigators (XY and JD) screened all studies carefully to ensure that they met the inclusion criteria. Inconsistencies were resolved by the chief investigator GPJ if they existed.

\section{Quality assessment}

The quality of the included studies was assessed by using an 11-item checklist for cross-sectional study quality, as recommended by the Agency for Healthcare Research and Quality (AHRQ) [26]. For the 11-item checklist, an item would be scored '0' if an answer of "NO" or "UNCLEAR" was given; if an answer of "YES" was given, then the item scored ' 1 '. The definition of article quality was as follows: low quality $=0-3$; moderate quality $=4-7$; and high quality $=8-11$ (S3 and S4 Tables).

\section{Statistical analysis}

First, pooled ORs with 95\% CIs were calculated by using the extracted raw data, crude or adjusted ORs with $95 \%$ CIs that evaluate the strength of the association between fertility desire/intention among PLHIV and the factors of interest (sex, age, marriage status, educational level, number of children and ART experience). Then six meta-analyses were performed. To assess the robustness of the outcomes, we conducted subgroup analysis in terms of publication year, region, and quality assessment score. Heterogeneity between the studies was assessed by using the Cochran's Q Chi-square test and $\mathrm{I}^{2}$ analysis ( $\mathrm{I}^{2}$ values of $50 \%$ and $75 \%$ were considered moderate and high heterogeneity respectively). When $\mathrm{I}^{2} \geq 50 \%$, we selected a random-effects model for heterogeneity analyses, and an otherwise fixed-effects model. Sensitivity analysis was performed to explore the impact of individual studies on the results. We evaluated publication bias through visual inspection of asymmetry in funnel plots or with the $\mathrm{P}$ value of Egger's test. We carried out all analyses were done with Stata (Version12.0).

\section{Results}

\section{Study selection}

According to the search strategy we retrieved 5,314 articles, of which 1,795 were duplicates. Finally, 50 articles were assessed for eligibility in these meta-analyses after full-text screening $[10,14,27-74]$. A flowchart of the literature search was presented in Fig 1.

\section{Description of the studies}

In these meta-analyses, we included a total of 50 articles with 22,367 participants. All included studies were cross-sectional and published between 2000 and -2019, and scored no less than 6 points on the basis of quality assessment. According to the income group defined by the World Bank for 2017, the study countries were categorized as low income, lower middle income, upper middle income, and high income [75]. Among these studies, 20 were conducted in low-income countries (LICs), 12 in lower middle-income countries (LMICs), 11 in upper middle-income countries (UMICs) and 7 in high-income countries (HICs). The general information is shown in Table 1.

\section{Meta-analysis of fertility desire}

The prevalence of fertility desire among PLHIV was $13.57 \%$ to $80.00 \%$ in the fifty included studies (Table 1). The meta-analysis revealed that the prevalence of fertility desire was $42.04 \%$ (95\% CI: $37.80,46.28 \%)$, with high heterogeneity $\left(I^{2}=97.9 \%, P<0.001\right)$ using the randomeffects model (Fig 2). 


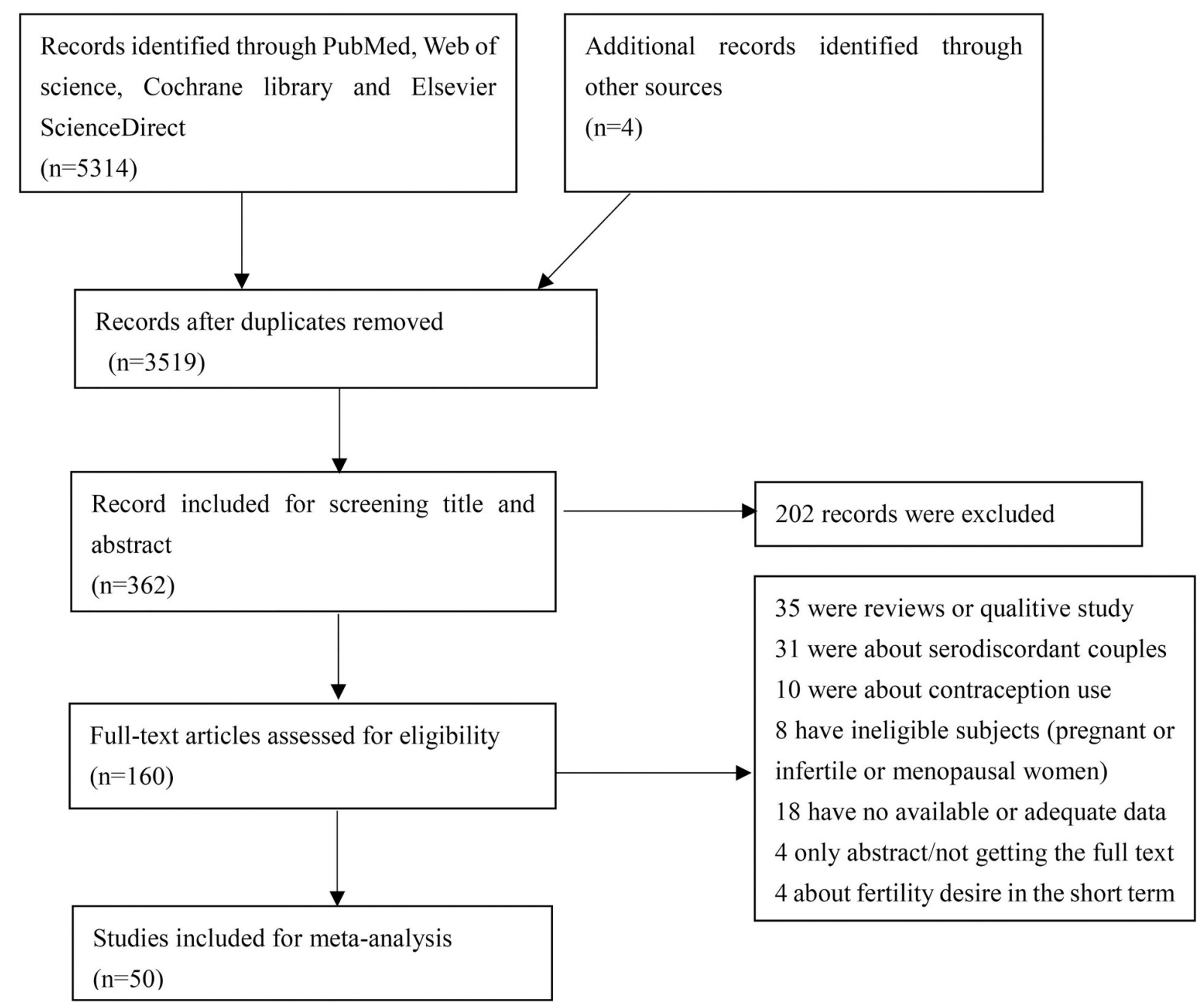

Fig 1. Flowchart of the literature search.

https://doi.org/10.1371/journal.pone.0248872.g001

We have reported the meta-analyses of the associated factors. As portrayed in Fig 3, in this meta-analysis, we have included 26 studies, five of which showed a statistically significant association between ART and fertility desire/intention. As a result, the pooled OR indicated that the fertility desire of PLHIV is statistically and significantly associated with ART (OR = 1.11; 95\% CI:1.00-1.23; $P=0.043$ ). The testing for heterogeneity did not reveal variability among the included studies $\left(I^{2}=44.1 \%, P=0.009\right)$.

As presented in Fig 4, twenty-five studies were included in this meta-analysis. Thirteen studies showed statistically significant association between sex and fertility desire/intention. Eleven suggested that men have more fertility desire than women while the other two implied the opposite. In terms of sex, the overall OR indicated that men have higher fertility desire than women $(\mathrm{OR}=1.51 ; 95 \% \mathrm{CI}: 1.10-2.09)$. It should be noted that heterogeneity among the included studies was high $\left(I^{2}=90.7 \%, P<0.001\right)$.

As seen in Fig 5, we included fourteen studies in the meta-analysis of the association between fertility desire and age. It is evident that being younger than 30 is a strong predictor of fertility desire among PLHIV, as almost all ORs with 95\% CIs of the included studies fell on 
Table 1. The main features of the studies included in the meta-analysis.

\begin{tabular}{|c|c|c|c|c|c|c|c|c|}
\hline First author & $\begin{array}{c}\text { Publication } \\
\text { year }\end{array}$ & Location & $\begin{array}{c}\text { Country's income } \\
\text { level }\end{array}$ & Study design & $\begin{array}{l}\text { Sample } \\
\text { size }\end{array}$ & $\begin{array}{c}\text { Number of men/ } \\
\text { women }\end{array}$ & $\begin{array}{c}\text { Fertility desire } \\
\text { (\%) }\end{array}$ & $\begin{array}{c}\text { Study quality } \\
\text { score }\end{array}$ \\
\hline Abbawa, F. & 2015 & Ethiopia & Low & $\begin{array}{c}\text { cross- } \\
\text { sectional }\end{array}$ & 422 & $217 / 205$ & $141(33.41 \%)$ & 7 \\
\hline Adilo, T. M. & 2017 & Ethiopia & Low & $\begin{array}{c}\text { cross- } \\
\text { sectional }\end{array}$ & 416 & $124 / 292$ & $227(54.57 \%)$ & 7 \\
\hline Adler, D. H. & 2017 & $\begin{array}{l}\text { South } \\
\text { Africa }\end{array}$ & Upper middle & $\begin{array}{c}\text { cross- } \\
\text { sectional }\end{array}$ & 50 & $0 / 50$ & $40(80.00 \%)$ & 7 \\
\hline Alemayehu B & 2012 & Ethiopia & Low & $\begin{array}{c}\text { cross- } \\
\text { sectional }\end{array}$ & 307 & $185 / 122$ & $203(66.12 \%)$ & 7 \\
\hline Asfaw, H. M. & 2014 & Ethiopia & Low & $\begin{array}{c}\text { cross- } \\
\text { sectional }\end{array}$ & 1855 & $0 / 1855$ & $815(43.94 \%)$ & 6 \\
\hline Cohn, S. E. & 2018 & USA & High & $\begin{array}{c}\text { cross- } \\
\text { sectional }\end{array}$ & 1425 & $1181 / 244$ & $580(40.70 \%)$ & 8 \\
\hline Cooper, D. & 2009 & $\begin{array}{l}\text { South } \\
\text { Africa }\end{array}$ & Upper middle & $\begin{array}{c}\text { cross- } \\
\text { sectional }\end{array}$ & 459 & $174 / 285$ & $148(32.24 \%)$ & 9 \\
\hline de Souza, M. R. & 2017 & Brazil & Upper middle & $\begin{array}{c}\text { cross- } \\
\text { sectional }\end{array}$ & 274 & $0 / 274$ & $71(25.91 \%)$ & 7 \\
\hline Demissie, D. B. & 2014 & Ethiopia & Low & $\begin{array}{c}\text { cross- } \\
\text { sectional }\end{array}$ & 340 & $126 / 214$ & $133(39.12 \%)$ & 7 \\
\hline $\begin{array}{c}\text { Finocchario Kessler, } \\
\text { S.1 }\end{array}$ & 2010 & USA & High & $\begin{array}{c}\text { cross- } \\
\text { sectional }\end{array}$ & 181 & $0 / 181$ & $107(59.12 \%)$ & 8 \\
\hline $\begin{array}{c}\text { Finocchario Kessler, } \\
\text { S.2 }\end{array}$ & 2014 & Brazil & Upper middle & $\begin{array}{c}\text { cross- } \\
\text { sectional }\end{array}$ & 295 & $295 / 0$ & $115(38.98 \%)$ & 8 \\
\hline Erhabor, O. & 2012 & Nigeria & Lower middle & $\begin{array}{c}\text { cross- } \\
\text { sectional }\end{array}$ & 195 & $88 / 107$ & $111(56.92 \%)$ & 6 \\
\hline Gyimah, A. A. & 2015 & Ghana & Lower middle & $\begin{array}{c}\text { cross- } \\
\text { sectional }\end{array}$ & 295 & $0 / 295$ & $172(58.31 \%)$ & 8 \\
\hline Haddad, L. B. & 2016 & USA & High & $\begin{array}{c}\text { cross- } \\
\text { sectional }\end{array}$ & 181 & $0 / 181$ & $62(34.25 \%)$ & 7 \\
\hline Heard, I. & 2007 & France & High & $\begin{array}{c}\text { cross- } \\
\text { sectional }\end{array}$ & 1254 & $699 / 555$ & $322(25.68 \%)$ & 9 \\
\hline Hernando, V. & 2014 & Spain & High & $\begin{array}{c}\text { cross- } \\
\text { sectional }\end{array}$ & 134 & $0 / 134$ & $66(49.25 \%)$ & 9 \\
\hline Iliyasu, Z. & 2009 & Nigeria & Lower middle & $\begin{array}{c}\text { cross- } \\
\text { sectional }\end{array}$ & 340 & $85 / 255$ & $219(64.41 \%)$ & 8 \\
\hline Jose, $\mathrm{H}$. & 2016 & India & Lower middle & $\begin{array}{c}\text { cross- } \\
\text { sectional }\end{array}$ & 230 & $132 / 98$ & $77(33.48 \%)$ & 8 \\
\hline Kaida, A. & 2011 & $\begin{array}{l}\text { South } \\
\text { Africa }\end{array}$ & Upper middle & $\begin{array}{c}\text { cross- } \\
\text { sectional }\end{array}$ & 432 & $0 / 432$ & $130(30.09 \%)$ & 9 \\
\hline Kawale, P. & 2014 & Malawi & Low & $\begin{array}{c}\text { cross- } \\
\text { sectional }\end{array}$ & 202 & $75 / 127$ & $103(50.99 \%)$ & 9 \\
\hline Kipp, W. & 2011 & Uganda & Low & $\begin{array}{c}\text { cross- } \\
\text { sectional }\end{array}$ & 199 & $77 / 122$ & $27(13.57 \%)$ & 9 \\
\hline Krashin, J. W. & 2018 & Malawi & Low & $\begin{array}{c}\text { cross- } \\
\text { sectional }\end{array}$ & 558 & $250 / 308$ & $175(31.36 \%)$ & 8 \\
\hline Laar, A. K. & 2015 & Ghana & Lower middle & $\begin{array}{c}\text { cross- } \\
\text { sectional }\end{array}$ & 318 & $0 / 318$ & $135(42.45 \%)$ & 7 \\
\hline Laryea, D. O. & 2014 & Ghana & Lower middle & $\begin{array}{l}\text { cross- } \\
\text { sectional }\end{array}$ & 230 & $0 / 230$ & $123(53.48 \%)$ & 7 \\
\hline Litwin, L. E. & 2015 & Uganda & Low & $\begin{array}{c}\text { cross- } \\
\text { sectional }\end{array}$ & 436 & $0 / 436$ & $162(37.16 \%)$ & 9 \\
\hline Maier, M. & 2009 & Uganda & Low & $\begin{array}{l}\text { cross- } \\
\text { sectional }\end{array}$ & 501 & $0 / 501$ & $73(14.57 \%)$ & 9 \\
\hline
\end{tabular}


Table 1. (Continued)

\begin{tabular}{|c|c|c|c|c|c|c|c|c|}
\hline First author & $\begin{array}{c}\text { Publication } \\
\text { year }\end{array}$ & Location & $\begin{array}{c}\text { Country's income } \\
\text { level }\end{array}$ & Study design & $\begin{array}{l}\text { Sample } \\
\text { size }\end{array}$ & $\begin{array}{c}\text { Number of men/ } \\
\text { women }\end{array}$ & $\begin{array}{c}\text { Fertility desire } \\
(\%)\end{array}$ & $\begin{array}{c}\text { Study quality } \\
\text { score }\end{array}$ \\
\hline Mayhew, S. H. & 2017 & Kenya & Lower middle & $\begin{array}{c}\text { cross- } \\
\text { sectional }\end{array}$ & 234 & $0 / 234$ & $66(28.21 \%)$ & 8 \\
\hline Mekonnen, B. & 2019 & Ethiopia & Low & $\begin{array}{c}\text { cross- } \\
\text { sectional }\end{array}$ & 427 & $0 / 427$ & $172(40.28 \%)$ & 9 \\
\hline Mekonnen, H. & 2017 & Ethiopia & Low & $\begin{array}{c}\text { cross- } \\
\text { sectional }\end{array}$ & 360 & $0 / 360$ & $174(48.33 \%)$ & 8 \\
\hline Melaku, Y. A. & 2014 & Ethiopia & Low & $\begin{array}{c}\text { cross- } \\
\text { sectional }\end{array}$ & 964 & $0 / 964$ & $439(45.54 \%)$ & 9 \\
\hline Mmbaga, E. J. & 2013 & Tanzania & Lower middle & $\begin{array}{c}\text { cross- } \\
\text { sectional }\end{array}$ & 410 & $146 / 264$ & $152(37.07 \%)$ & 7 \\
\hline Moyo, W. & 2004 & Zimbabwe & Lower middle & $\begin{array}{c}\text { cross- } \\
\text { sectional }\end{array}$ & 2250 & $0 / 2250$ & $1210(53.78 \%)$ & 8 \\
\hline Myer, L. & 2007 & $\begin{array}{l}\text { South } \\
\text { Africa }\end{array}$ & Upper middle & $\begin{array}{c}\text { cross- } \\
\text { sectional }\end{array}$ & 311 & $84 / 227$ & $89(28.62 \%)$ & 8 \\
\hline Nakayiwa, S. & 2006 & Uganda & Low & $\begin{array}{c}\text { cross- } \\
\text { sectional }\end{array}$ & 1092 & $488 / 604$ & $174(15.93 \%)$ & 8 \\
\hline Nedjat, S. & 2015 & Iran & Upper middle & $\begin{array}{c}\text { cross- } \\
\text { sectional }\end{array}$ & 400 & $240 / 160$ & $161(40.25 \%)$ & 7 \\
\hline Nobrega, A. A. & 2007 & Brazil & Upper middle & $\begin{array}{c}\text { cross- } \\
\text { sectional }\end{array}$ & 229 & $0 / 229$ & $91(39.74 \%)$ & 7 \\
\hline $\begin{array}{c}\text { Okome-Nkoumou, } \\
\text { M. }\end{array}$ & 2015 & Gabon & Upper middle & $\begin{array}{c}\text { cross- } \\
\text { sectional }\end{array}$ & 422 & $85 / 337$ & $329(77.96 \%)$ & 6 \\
\hline Oladapo, O. T. & 2005 & Nigeria & Lower middle & $\begin{array}{c}\text { cross- } \\
\text { sectional }\end{array}$ & 147 & $52 / 95$ & $93(63.27 \%)$ & 9 \\
\hline Paiva, V. & 2007 & Brazil & Upper middle & $\begin{array}{c}\text { cross- } \\
\text { sectional }\end{array}$ & 739 & $206 / 533$ & $202(27.33 \%)$ & 7 \\
\hline Pokharel, R. & 2018 & Nepal & Lower middle & $\begin{array}{c}\text { cross- } \\
\text { sectional }\end{array}$ & 252 & $131 / 121$ & $51(20.24 \%)$ & 7 \\
\hline Pottinger, A. M. & 2019 & Jamaica & Upper middle & $\begin{array}{c}\text { cross- } \\
\text { sectional }\end{array}$ & 251 & $121 / 130$ & $166(66.14 \%)$ & 7 \\
\hline Rhodes, C. M. & 2016 & USA & High & $\begin{array}{c}\text { cross- } \\
\text { sectional }\end{array}$ & 100 & $0 / 100$ & $44(44.00 \%)$ & 8 \\
\hline Shiferaw, $\mathrm{T}$. & 2019 & Ethiopia & Low & $\begin{array}{c}\text { cross- } \\
\text { sectional }\end{array}$ & 374 & $0 / 374$ & $175(46.79 \%)$ & 9 \\
\hline Sufa, A. & 2014 & Ethiopia & Low & $\begin{array}{c}\text { cross- } \\
\text { sectional }\end{array}$ & 456 & $0 / 421$ & $192(42.11 \%)$ & 7 \\
\hline Tamene, W. & 2007 & Ethiopia & Low & $\begin{array}{c}\text { cross- } \\
\text { sectional }\end{array}$ & 460 & $216 / 244$ & $185(40.22 \%)$ & 6 \\
\hline Tesfaye L & 2012 & Ethiopia & Low & $\begin{array}{c}\text { cross- } \\
\text { sectional }\end{array}$ & 389 & $171 / 218$ & $164(42.16 \%)$ & 8 \\
\hline Thomson, K. A. & 2018 & USA & High & $\begin{array}{c}\text { cross- } \\
\text { sectional }\end{array}$ & 100 & $72 / 28$ & $43(43.00 \%)$ & 7 \\
\hline Wagner, G. & 2012 & Uganda & Low & $\begin{array}{c}\text { cross- } \\
\text { sectional }\end{array}$ & 233 & $94 / 139$ & $103(44.21 \%)$ & 8 \\
\hline Wagner, G. J. & 2013 & Uganda & Low & $\begin{array}{c}\text { cross- } \\
\text { sectional }\end{array}$ & 767 & $261 / 506$ & $237(30.90 \%)$ & 8 \\
\hline Wekesa, E. & 2014 & Kenya & Lower middle & $\begin{array}{c}\text { cross- } \\
\text { sectional }\end{array}$ & 463 & $193 / 270$ & $157(33.91 \%)$ & 9 \\
\hline
\end{tabular}

the side of increased fertility desire. Similarly, the pooled OR with moderate heterogeneity $\left(I^{2}=51.0 \%, P=0.014\right)$, showed that PLHIV younger than 30 years have a 2.6 -fold increase in fertility desire compared to their older counterparts $(\mathrm{OR}=2.65$; $95 \%$ CI:2.24-3.14) 


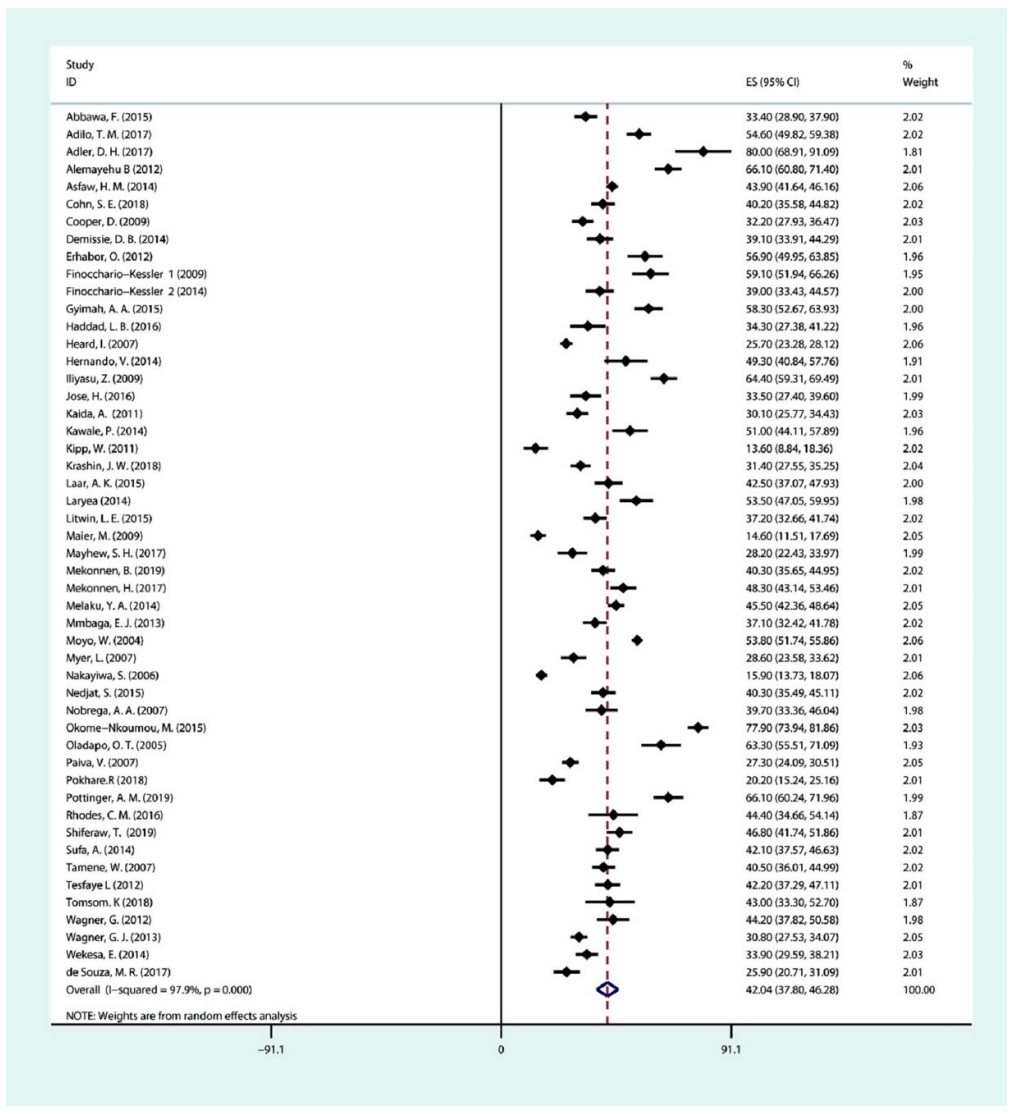

Fig 2. Forest plot of the overall prevalence of fertility desire among people living with HIV.

https://doi.org/10.1371/journal.pone.0248872.g002

As outlined in Fig 6, a meta-analysis including 24 studies was performed to assess association between marriage status and fertility desire. Among the 11 studies that revealed a significant association, only one indicated that PLHIV who are married/cohabiting have less fertility desire than PLHIV who are not married (single, widowed, divorced, or separated). The overall OR demonstrated a positive association of fertility desire with being married/cohabiting ( $\mathrm{OR}=1.34$; $95 \%$ CI:1.08-1.65). However, we noted the high heterogeneity $\left(I^{2}=83.8 \%, P<0.001\right)$.

As portrayed in Fig 7, we included twenty-two studies in the meta-analysis. Five studies consistently found that educational level is associated with fertility desire among PLHIV. The pooled OR implied that, with moderate heterogeneity among the included studies $\left(I^{2}=69.8 \%\right.$, $P=<0.001)$, PLHIV with a level of up to primary education have less fertility desire than PLHIV whose educational level is secondary or above ( $\mathrm{OR}=0.85$; 95\% CI: $0.73-1.00$ ).

As displayed in Fig 8, there were 26 included studies, of which only four showed no significant association between the number of children and fertility desire in the meta-analysis. As a result, having no children is another strong predictor of fertility desire among PLHIV. The pooled OR indicated that PLHIV who are childless have a nearly 4 -fold increase in fertility than PLHIV who have one or more children ( $\mathrm{OR}=3.99$; 95\% CI:3.06-5.20). There was high heterogeneity among the included studies $\left(I^{2}=84.9 \%, P<0.001\right)$.

\section{Subgroup analysis}

Next, we performed subgroup analysis on the basis of publication year, the region where the study was done, and the quality assessment score of the studies. We found associations of 


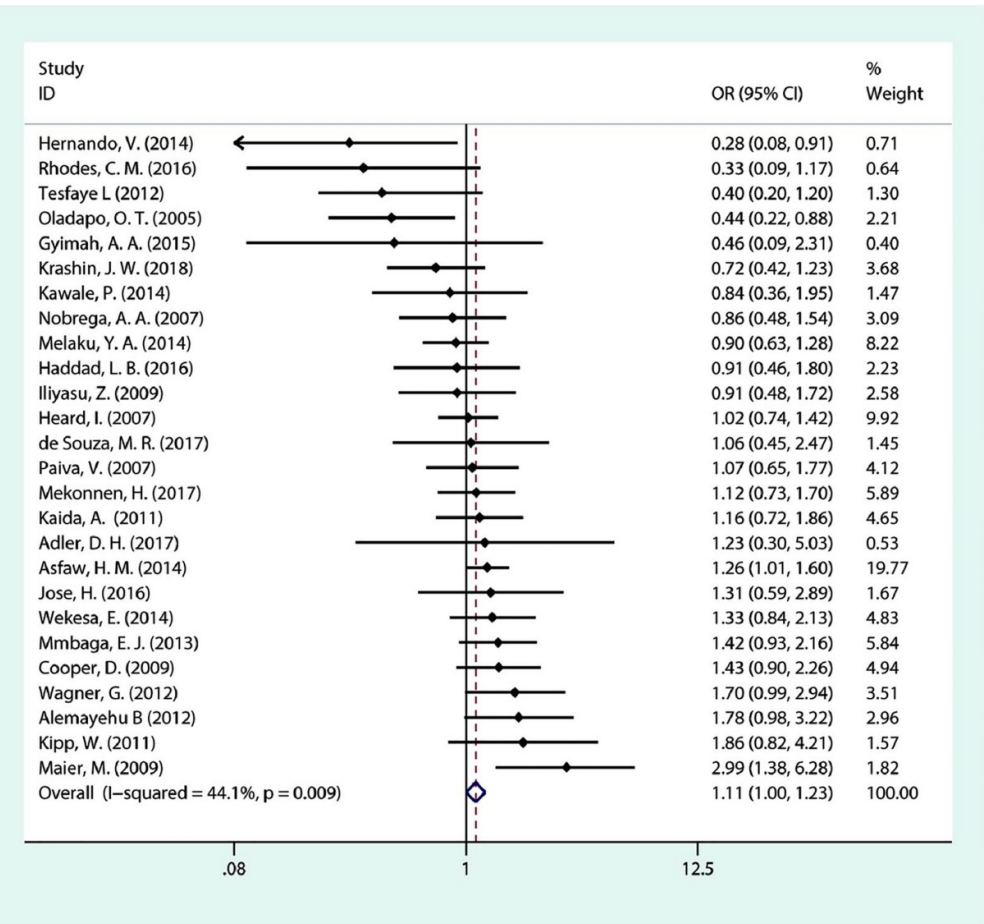

Fig 3. Forest plot of pooled OR for fertility desire in PLHIV (ART experienced vs ART naive).

https://doi.org/10.1371/journal.pone.0248872.g003

fertility desire with age and with the number of children in all subgroups that people living with HIV who were younger than 30 and had no children experienced greater fertility desire. There were relationships between sex and fertility desire shown in studies from Africa

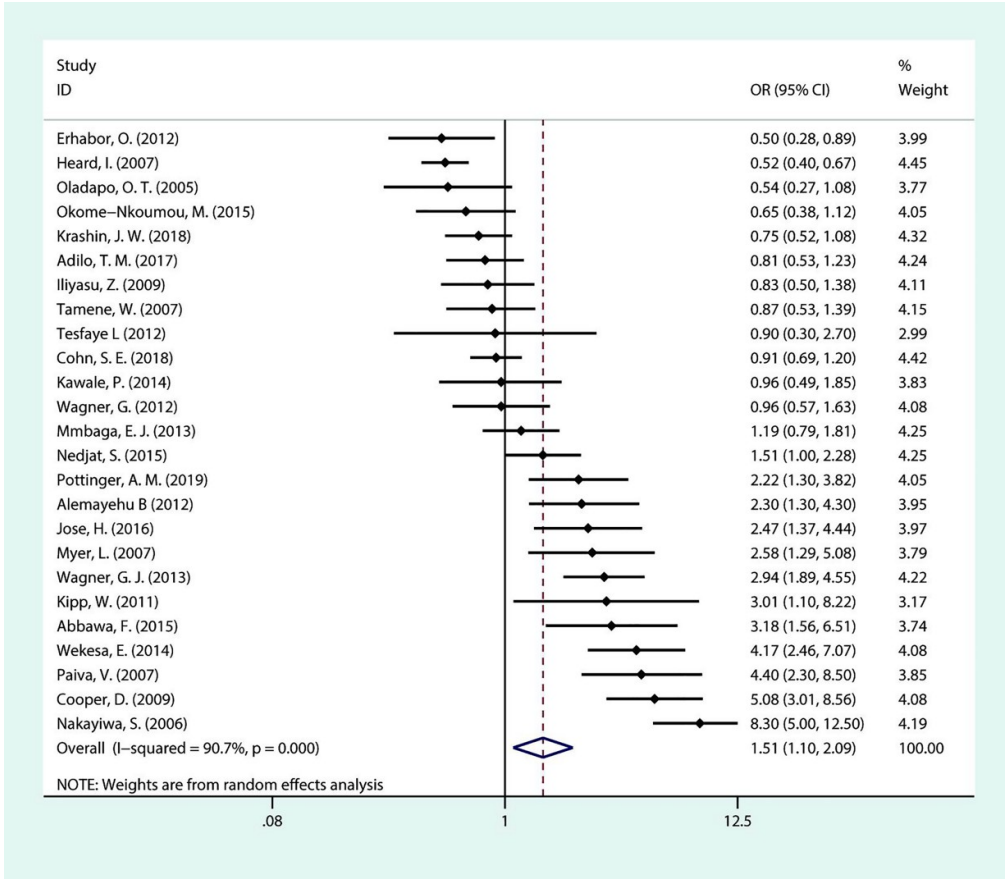

Fig 4. Forest plot of pooled OR for fertility desire in PLHIV (men vs women). 


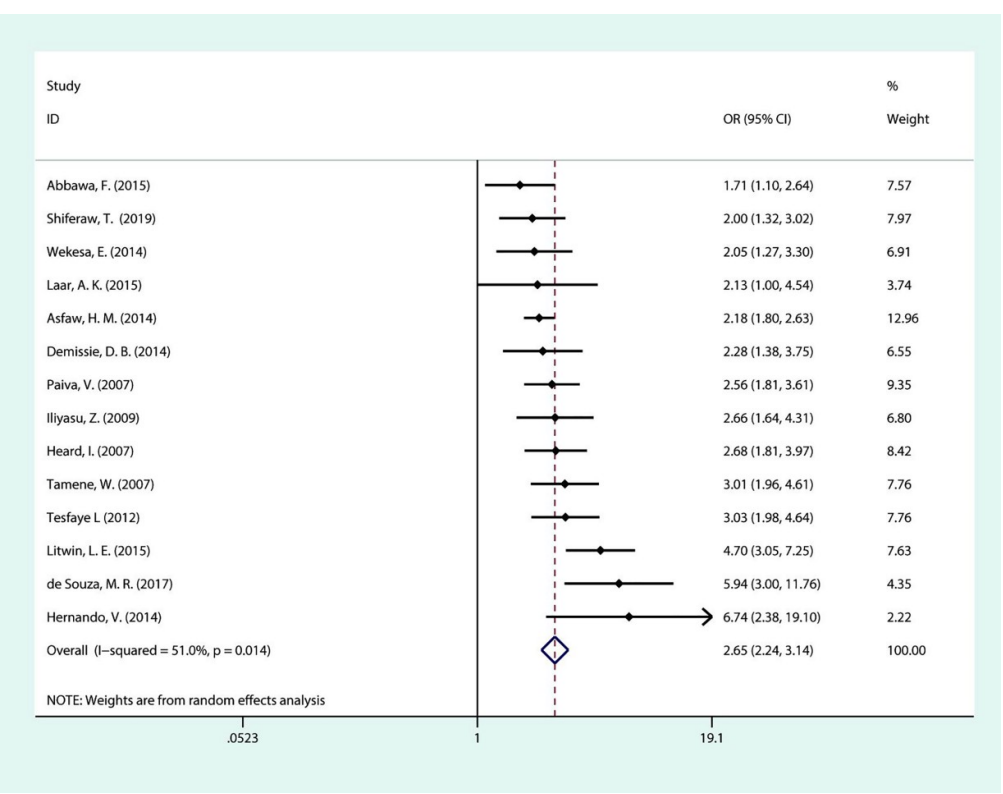

Fig 5. Forest plot of pooled OR for fertility desire in PLHIV (aged below 30 years vs aged 30 and above).

https://doi.org/10.1371/journal.pone.0248872.g005

$\left(\mathrm{OR}=1.54 ; 95 \% \mathrm{CI}: 1.04-2.30 ; I^{2}=90.3 \%\right)$. In the subgroup of marriage status, studies published after $2014\left(\mathrm{OR}=1.75 ; 95 \% \mathrm{CI}: 1.44-2.12 ; I^{2}=63.6\right)$, those conducted in Africa $\left(\mathrm{OR}=1.42 ; 95 \%\right.$ CI: $\left.1.13-1.77 ; I^{2}=83.9 \%\right)$ and those that scored higher than 7 on the quality assessment $\left(\mathrm{OR}=1.44 ; 95 \%\right.$ CI: $\left.1.02-2.03 ; I^{2}=87.8 \%\right)$ were statistically significant. In light of educational level, we observed an association with fertility desire in studies from Africa as well

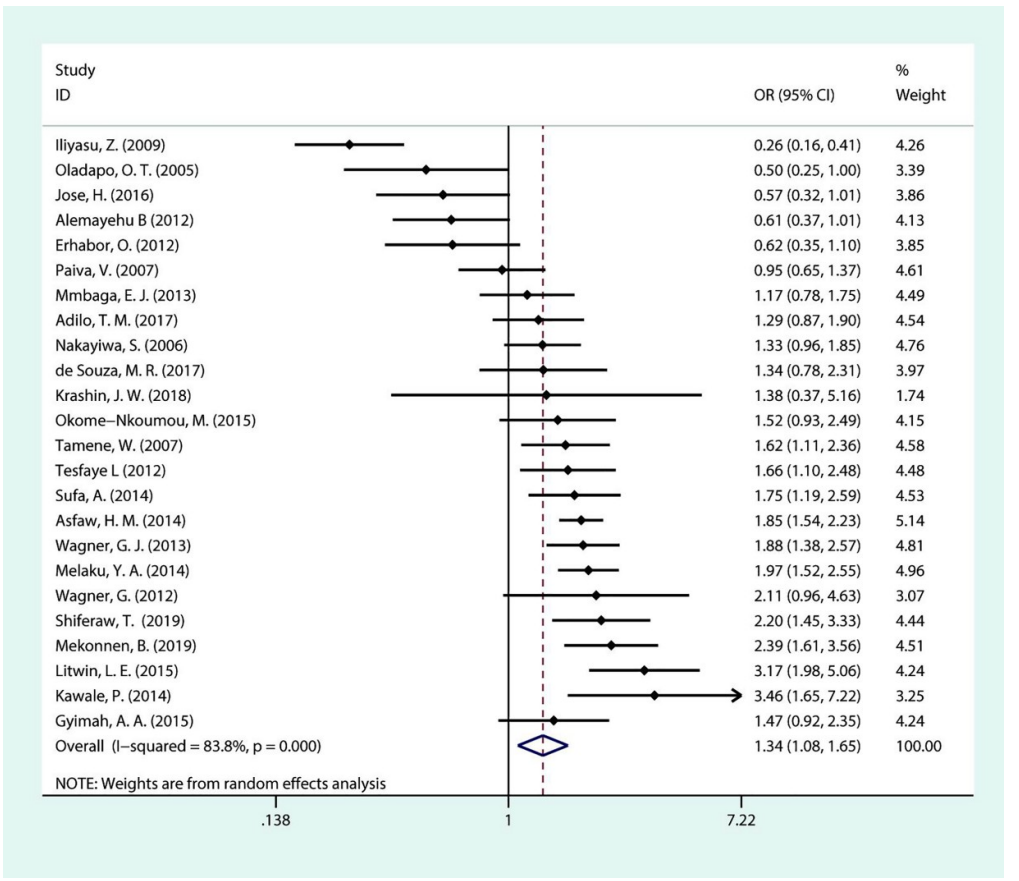

Fig 6. Forest plot of pooled OR for fertility desire in PLHIV (currently married vs unmarried). 


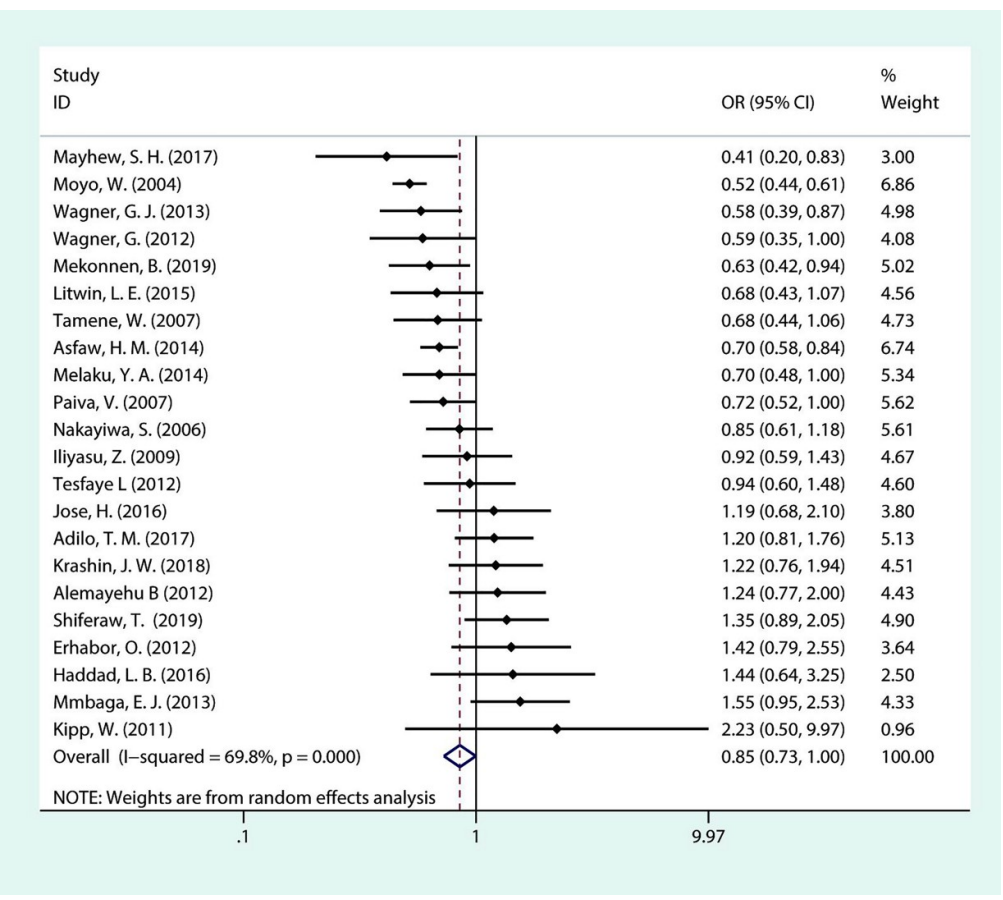

Fig 7. Forest plot of pooled OR for fertility desire in PLHIV (up to primary vs secondary or above).

https://doi.org/10.1371/journal.pone.0248872.g007

as in which the studies of which quality assessment score was higher than 7 , and the pooled ORs were 0.84 (95\% CI: 0.72-0.99, $I^{2}=72.2 \%$ ) and 0.78 (95\% CI: $\left.0.64-0.95, I^{2}=68.3 \%\right)$ respectively. The details of the subgroup analysis were summarized in Table 2.

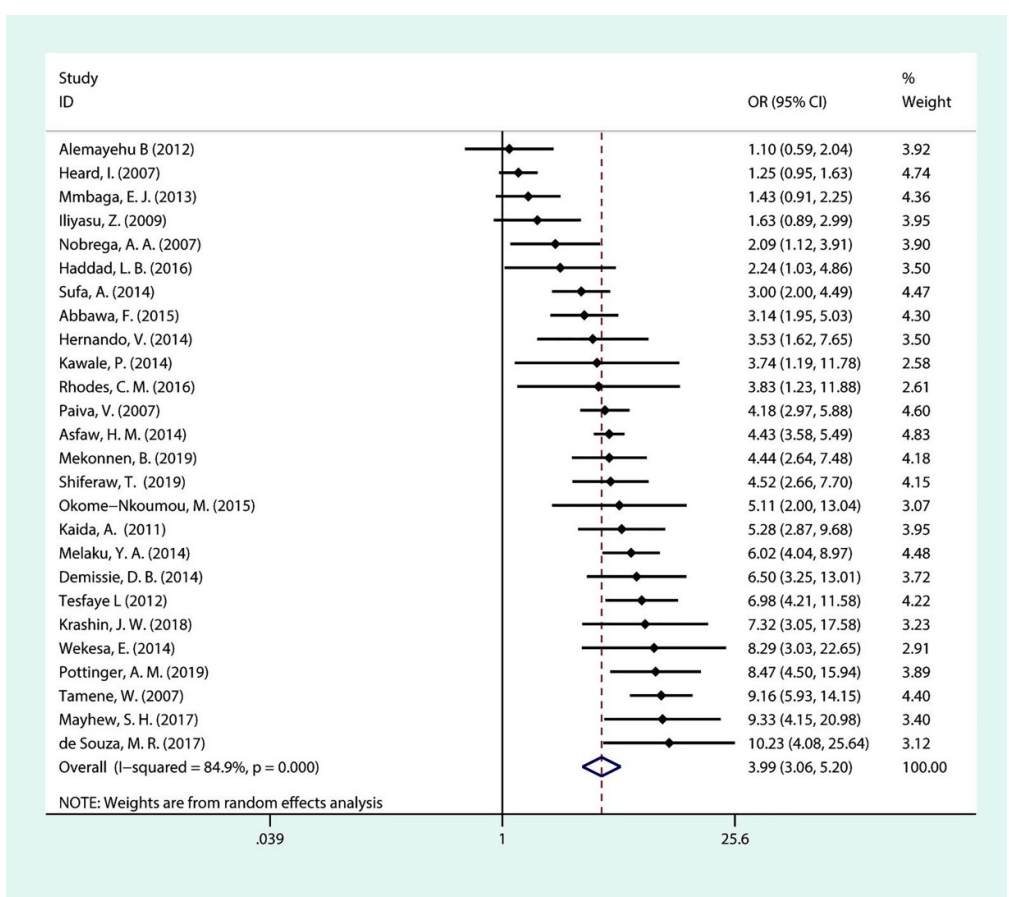

Fig 8. Forest plot of pooled OR for fertility desire in PLHIV (having no children vs having one or more children). 
Table 2. The results of the meta-analysis, the heterogeneity test, and publication bias.

\begin{tabular}{|c|c|c|c|c|c|}
\hline Subgroup $^{\text {a }}$ & Number of studies & OR $(95 \% \mathrm{CI})$ & I square (\%) & $\mathbf{P}$ & P for Egger's test \\
\hline \multicolumn{6}{|l|}{ ART } \\
\hline Overall & 26 & $1.11(1.00-1.23)^{\mathbf{b}}$ & 44.1 & 0.009 & 0.109 \\
\hline \multicolumn{6}{|l|}{ Publication year } \\
\hline Pre $2014^{c}$ & 13 & $1.17(0.93-1.48)$ & 57.0 & 0.006 & 0.187 \\
\hline Post 2014 & 13 & $1.05(0.91-1.22)$ & 23.8 & 0.203 & 0.469 \\
\hline \multicolumn{6}{|l|}{ Region } \\
\hline Africa & 18 & $1.14(0.95-1.37)$ & 50.1 & 0.008 & 0.457 \\
\hline Other & 8 & $0.95(0.77-1.17)$ & 10.3 & 0.350 & 0.089 \\
\hline \multicolumn{6}{|l|}{ Quality assessment score } \\
\hline$\leq 7$ & 8 & $1.22(1.04-1.44)$ & 0.0 & 0.717 & 0.014 \\
\hline$>7$ & 18 & $1.01(0.81-1.25)$ & 55.2 & 0.003 & 0.964 \\
\hline \multicolumn{6}{|l|}{ Sex } \\
\hline Overall & 25 & $1.51(1.10-2.09)$ & 90.7 & $<0.001$ & 0.038 \\
\hline \multicolumn{6}{|l|}{ Publication year } \\
\hline Pre 2014 & 15 & $1.58(0.96-2.62)$ & 92.9 & $<0.001$ & 0.183 \\
\hline Post 2014 & 10 & $1.40(0.96-2.05)$ & 84.8 & $<0.001$ & 0.096 \\
\hline \multicolumn{6}{|l|}{ Region } \\
\hline Africa & 19 & $1.54(1.04-2.30)$ & 90.3 & $<0.001$ & 0.696 \\
\hline Other & 6 & $1.41(0.82-2.42)$ & 90.7 & $<0.001$ & 0.008 \\
\hline \multicolumn{6}{|c|}{ Quality assessment score } \\
\hline$\leq 7$ & 10 & $1.37(0.93-2.02)$ & 82.5 & $<0.001$ & 0.187 \\
\hline$>7$ & 15 & $1.61(1.00-2.59)$ & 93.2 & $<0.001$ & 0.108 \\
\hline \multicolumn{6}{|l|}{ Age } \\
\hline Overall & 14 & $2.65(2.24-3.14)$ & 51.0 & 0.014 & 0.082 \\
\hline \multicolumn{6}{|l|}{ Publication year } \\
\hline Pre 2014 & 5 & $2.76(2.30-3.32)$ & 0.0 & 0.967 & 0.38 \\
\hline Post 2014 & 9 & $2.64(2.02-3.47)$ & 67.2 & 0.002 & 1.37 \\
\hline \multicolumn{6}{|l|}{ Region } \\
\hline Africa & 10 & $2.41(2.14-2.71)$ & 44.7 & 0.061 & 0.527 \\
\hline Other & 4 & $3.47(2.28-5.26)$ & 58.8 & 0.063 & 0.054 \\
\hline \multicolumn{6}{|c|}{ Quality assessment score } \\
\hline$\leq 7$ & 7 & $2.34(2.04-2.68)$ & 47.0 & 0.079 & 0.329 \\
\hline$>7$ & 7 & $2.87(2.21-3.73)$ & 52.3 & 0.049 & 0.343 \\
\hline \multicolumn{6}{|l|}{ Marital status } \\
\hline Overall & 24 & $1.34(1.08-1.65)$ & 83.8 & $<0.001$ & 0.115 \\
\hline \multicolumn{6}{|l|}{ Publication year } \\
\hline Pre 2014 & 11 & $0.99(0.69-1.42)$ & 86.8 & $<0.001$ & 0.161 \\
\hline Post 2014 & 13 & $1.75(1.44-2.12)$ & 63.6 & 0.001 & 0.653 \\
\hline \multicolumn{6}{|l|}{ Region } \\
\hline Africa & 21 & $1.42(1.13-1.77)$ & 83.9 & $<0.001$ & 0.198 \\
\hline Other & 3 & $0.91(0.60-1.39)$ & 56.2 & 0.102 & 0.878 \\
\hline \multicolumn{6}{|c|}{ Quality assessment score } \\
\hline$\leq 7$ & 10 & $1.23(0.97-1.57)$ & 73.7 & $<0.001$ & 0.02 \\
\hline$>7$ & 14 & $1.44(1.02-2.03)$ & 87.8 & $<0.001$ & 0.483 \\
\hline \multicolumn{6}{|l|}{ Educational level } \\
\hline Overall & 22 & $0.85(0.73-1.00)^{\mathbf{d}}$ & 69.8 & $<0.001$ & 0.003 \\
\hline Publication year & & & & & \\
\hline
\end{tabular}


Table 2. (Continued)

\begin{tabular}{|c|c|c|c|c|c|}
\hline Subgroup $^{a}$ & Number of studies & OR $(95 \% \mathrm{CI})$ & I square (\%) & $\mathbf{P}$ & $P$ for Egger's test \\
\hline Pre 2014 & 12 & $0.84(0.67-1.06)$ & 73.3 & $<0.001$ & 0.003 \\
\hline Post 2014 & 10 & $0.87(0.70-1.09)$ & 64.3 & 0.003 & 0.288 \\
\hline \multicolumn{6}{|l|}{ Region } \\
\hline Africa & 19 & $0.84(0.72-0.99)$ & 72.2 & $<0.001$ & 0.01 \\
\hline Other & 3 & $0.87(0.68-1.14)$ & 48.5 & 0.143 & 0.146 \\
\hline \multicolumn{6}{|c|}{ Quality assessment score } \\
\hline$\leq 7$ & 8 & $0.99(0.78-1.29)$ & 68.2 & 0.003 & 0.019 \\
\hline$>7$ & 14 & $0.78(0.64-0.95)$ & 68.3 & $<0.001$ & 0.027 \\
\hline \multicolumn{6}{|l|}{ Number of child } \\
\hline Overall & 26 & $3.99(3.06-5.20)$ & 84.9 & $<0.001$ & 0.272 \\
\hline \multicolumn{6}{|l|}{ Publication year } \\
\hline Pre 2014 & 9 & $2.77(1.60-4.81)$ & 92.4 & $<0.001$ & 0.53 \\
\hline Post 2014 & 17 & $4.64(4.09-5.27)$ & 37.4 & 0.061 & 0.299 \\
\hline \multicolumn{6}{|l|}{ Region } \\
\hline Africa & 17 & $4.54(3.53-5.84)$ & 73.0 & $<0.001$ & 0.878 \\
\hline Other & 9 & $3.11(1.87-5.19)$ & 87.6 & $<0.001$ & 0.135 \\
\hline \multicolumn{6}{|c|}{ Quality assessment score } \\
\hline$\leq 7$ & 13 & $3.72(2.66-5.19)$ & 83.3 & $<0.001$ & 0.753 \\
\hline$>7$ & 13 & $4.33(2.76-6.80)$ & 86.7 & $<0.001$ & 0.034 \\
\hline
\end{tabular}

${ }^{a}:$ The variables in the subgroup analyses were ART (experienced vs naive), sex (men vs women), age (below 30 vs 30 and above), marital status (married/cohabiting vs not married), educational level (up to primary vs secondary or above), and number of children (none vs one child or more).

b, $\mathbf{d}: 1$ is not included in confidence intervals when the digits after decimal are kept three.

c: 2014 is the median.

\section{Heterogeneity analysis and publication bias}

The results of the heterogeneity test and Egger's test were outlined in Table 2. We performed the random-effects model to do meta-analyses for studies with heterogeneity $\left(I^{2} \geq 50 \%\right)$, and analyzed the rest using a fixed-effects model. According to Egger's test and the funnel plot, there was evidence of publication bias in many studies included in the meta-analyses of the association of fertility desire with sex and educational level. (Table 2, S1-S6 Figs)

\section{Discussion}

In our study, the prevalence of fertility desire among HIV-infected people was $42.04 \%$, which indicated that their desire to have children cannot be ignored. These meta-analyses demonstrate that for PLHIV, ART use, sex, age, marital status, number of children, and education level are all associated with fertility desire. However, many studies, including a meta-analysis, showed that ART use has no association with fertility desire among PLHIV [9, 24, 76, 77]. In effect, as the most efficient treatment for HIV-infected people, ART could improve their overall well-being, suppress the viral load to a great extent, and help them remain optimistic about fertility [42]. Therefore, it is reasonable for PLHIV on ART to have a higher prevalence of the desire for reproduction than their ART-naive counterparts. In contrast to the previous metaanalysis, this study found that HIV-infected men trend to have more fertility desire than HIVinfected women. Although men and women are both required in the reproductive process, women often suffer the most during pregnancy, which might make them more cautious about fertility [27]. In many patrilineal societies such as in South Africa, HIV-infected men's greater 
desire to have children than women might result from the inclination to leave something behind, such as lineage, after they pass away [78-80]. Younger PLHIV, as this study showed, have stronger fertility desire than their older counterparts. For one thing, the desire for children is usually strong in young people of reproductive age, irrespective of HIV infection status. For another, older HIV-infected persons might have already achieved their ideal family sizes and thus would not like to bear other children [14, 52]. Likewise, a majority of studies demonstrated that PLHIV with none or fewer children have a higher prevalence of fertility desire [11, $52,61]$. In some countries, such as Ethiopia, it is believed that family life will not be happy and fulfilled without a child [81]. Additionally, the desire to have biological children and a family of a certain size, as is case in many African nations, are significant reasons for fertility intention among HIV-infected women [82]. As this study indicated, PLHIV who are married or cohabiting have more fertility desire than HIV-infected people who are single, widowed, divorced or separated. Married HIV-infected individuals often have stable relationships or regular sexual partners, and thus might have more reliable support for raising children compared to PLHIV who are not married [50]. Within Ugandan society and in many cultures of Sub-Saharan Africa, childbearing plays a critical role in marriages and families, which may explain the greater fertility desire among PLHIV who are married than among those who are not [66]. Normally, better educated people are expected to have increasingly greater access to information, particularly, AIDS prevention knowledge such as the MTCT of HIV. In addition, welleducated people tend to have better jobs and relatively higher incomes which could make them have better access to medical services [83]. In terms of issues of reproduction, women with higher educational levels tend to make independent decisions, which may be related to higher fertility desire [15]. The meta-analysis likewise showed that HIV-infected people with higher educational levels have more fertility desire than their less educated peers.

Since the advent of ART, it has been possible for PLHIV to give birth to healthy children, due to an enhanced quality of life, longer lifespans and reduced MTCT [76]. However, as a predictor of reproductive practices, fertility desire, if increased, would prompt HIV-infected people to have unprotected intercourse, to give birth and to breastfeed their children, which might heighten the risk of infecting their partners, spouses or children with HIV [8, 84-86]. Many studies have revealed that in Africa horizontal and vertical transmission remain the primary forms of HIV infection, with transmission rates ranging from $20 \%-25 \%$ by HIV-positive people to their HIV-negative partners [87, 88]. Despite being aware of HIV transmission, For many HIV-infected individuals, strong fertility desire could pose barriers to contraception use and thus they would be at risk of unprotected sex [89]. Meanwhile, they would face a complex decision about whether to fulfill their desire to have children.

Consequently, it is important for local health care providers to develop appropriate reproductive health service policies and interventions for men and women living with HIV. For instance, HIV-infected individuals should be provided with counseling and related tests of HIV, such as tests of HIV viral load and CD4 count, and with advice on safe conception. Eligible HIV-infected individuals should initiate ART as soon as possible, and the use of assisted reproductive technology should be recommended for sero-discordant couples. On the whole, fully understanding the fertility desire of PLHIV is essential to providing targeted sexual and reproductive health services.

This study has many limitations. First, there were only six variables included. Previous studies have shown that the following factors are also related to fertility desire among PLHIV: health status, the duration of ART, the influence of partners or spouses, ethnicity, income, culture, stigma, and attitudes of local health care providers; we were unable to extracted these factors either due to a lack of or the unfitness of data for the meta-analyses [18]. As a consequence, it was not easy to draw conclusion on fertility desire by taking six limited 
variables as determinants. Further, it was found that the duration of ART has potential influence on fertility desire [33]. Therefore it seems more reasonable to consider duration of ART as a factor rather than ART use. Second, there might be a difference in the definition of educational status in various countries; as a result, the pooled effect size of the association between fertility desire and educational level might not be accurate. Moreover, both the lower limit (1.003) of CI in the analysis of ART and the upper limit (0.998) of CI in the analysis of educational level were close to 1 , which suggests the need for deeper investigation. Third, the fertility practices of PLHIV are affected by many social factors, such as discrimination; therefore studies on fertility desire might not reflect their genuine intentions. Fourth, the included studies in these meta-analyses were mainly from Sub-Saharan Africa and few were from high-income countries. Hence, positive association of fertility desire with the factors considered in this study is less likely to represent the countries in all of the included studies.

\section{Conclusion}

In summary, the results of this study demonstrate that fertility desire among PLHIV is associated with ART experience, sex, age, marital status, the number of children, and educational level. HIV-infected individuals who are on ART, are male, are younger than 30, are married/ cohabiting, are childless and have a secondary education or above have a higher prevalence of fertility desire. Through reproductive health counseling and care, further measures to prevent the horizontal and vertical transmission of HIV should be taken. In addition, their reproductive needs should be met, particularly targeted PLHIV.

\section{Supporting information}

S1 Table. PRISMA 2009 checklist.

(DOC)

S2 Table. Search for PubMed, Cochrane Library, Web of Science and ScienceDirect. (DOCX)

S3 Table. Scoring criteria for the quality of studies.

(XLSX)

S4 Table. Quality assessment of the included studies. (XLSX)

S1 Fig. Funnel plot of publication bias of the studies included in the analysis of the association of fertility desire with ART. (PNG)

S2 Fig. Funnel plot of publication bias of the studies included in the analysis of the association of fertility desire with sex.

$(\mathrm{PNG})$

S3 Fig. Funnel plot of publication bias of the studies included in the analysis of the association of fertility desire with age.

(PNG)

S4 Fig. Funnel plot of publication bias of the studies included in the analysis of the association of fertility desire with marital status.

$(\mathrm{PNG})$ 
S5 Fig. Funnel plot of publication bias of the studies included in the analysis of the association of fertility desire with educational level.

(PNG)

S6 Fig. Funnel plot of publication bias of the studies included in the analysis of the association of fertility desire with the number of children.

(PNG)

\section{Acknowledgments}

The authors would like to acknowledge the work of the research teams whose publications were included in this meta-analysis.

\section{Author Contributions}

Conceptualization: Xiang Yan.

Data curation: Jie Du.

Formal analysis: Xiang Yan.

Methodology: Xiang Yan.

Supervision: GuoPing Ji.

Writing - original draft: Xiang Yan.

Writing - review \& editing: GuoPing Ji.

\section{References}

1. UNAIDS. UNAIDS data 2019[R]. Geneva: UNAIDS Corporate Publications, 2019.

2. du Loû AD, Msellati P, Yao A, Noba V, Viho I, Ramon R, et al. Impaired fertility in HIV-1-infected pregnant women: a clinic-based survey in Abidjan, Côte d ,Ivoire, 1997. 1999; 13(4):517-21.

3. van Benthem BH, de Vincenzi I, Delmas $M-C$, Larsen $C$, van den Hoek $A$, Prins $M$, et al. Pregnancies before and after HIV diagnosis in a European cohort of HIV-infected women. 2000; 14(14):2171-8.

4. Lewis JJ, Ronsmans C, Ezeh A, Gregson SJA. The population impact of HIV on fertility in sub-Saharan Africa. 2004; 18:S35-S43. https://doi.org/10.1097/00002030-200406002-00005 PMID: 15319742

5. Hogg RS, O'Shaughnessy MV, Gataric N, Yip B, Craib K, Schechter MT, et al. Decline in deaths from AIDS due to new antiretrovirals. 1997; 349(9061):1294.

6. Hogg RS, Heath KV, Yip B, Craib KJ, O'shaughnessy MV, Schechter MT, et al. Improved survival among HIV-infected individuals following initiation of antiretroviral therapy. 1998; 279(6):450-4. https:// doi.org/10.1001/jama.279.6.450 PMID: 9466638

7. Kisakye P, Akena WO, Kaye DKJC, health, sexuality. Pregnancy decisions among HIV-positive pregnant women in Mulago Hospital, Uganda. 2010; 12(4):445-54.

8. Beyeza-Kashesya J, Ekstrom AM, Kaharuza F, Mirembe F, Neema S, Kulane AJBph. My partner wants a child: a cross-sectional study of the determinants of the desire for children among mutually disclosed sero-discordant couples receiving care in Uganda. 2010; 10(1):247. https://doi.org/10.1186/1471-245810-247 PMID: 20465794

9. Getachew M, Alemseged F, Abera M, Deribew AJEJoHD. Factors affecting fertility decisions of married men and women living with HIV in South Wollo Zone, Northeast Ethiopia. 2010; 24(3).

10. Sufa A, Wordofa MA, Wossen BA. Determinants of fertility intention among women living with hiv in western Ethiopia: implications for service delivery. Afr J Reprod Health. 2014; 18(4):54-60. Epub 2015/ 04/10. PMID: 25854093.

11. Loutfy MR, Hart TA, Mohammed SS, Su D, Ralph ED, Walmsley SL, et al. Fertility desires and intentions of HIV-positive women of reproductive age in Ontario, Canada: a cross-sectional study. 2009; 4 (12):e7925. https://doi.org/10.1371/journal.pone.0007925 PMID: 19997556

12. Loutfy MR, Hart TA, Mohammed SS, Su D, Ralph ED, Walmsley SL, et al. Fertility desires and intentions of HIV-positive women of reproductive age in Ontario, Canada: a cross-sectional study. PLoS 
One. 2009; 4(12):e7925. Epub 2009/12/10. https://doi.org/10.1371/journal.pone.0007925 PMID: 19997556; PubMed Central PMCID: PMC2785467.

13. Beyeza-Kashesya J, Ekstrom AM, Kaharuza F, Mirembe F, Neema S, Kulane A. My partner wants a child: a cross-sectional study of the determinants of the desire for children among mutually disclosed sero-discordant couples receiving care in Uganda. BMC Public Health. 2010; 10:247. Epub 2010/05/15 https://doi.org/10.1186/1471-2458-10-247 PMID: 20465794; PubMed Central PMCID: PMC2877675.

14. Mmbaga EJ, Leyna GH, Ezekiel MJ, Kakoko DC. Fertility desire and intention of people living with HIV/ AIDS in Tanzania: a call for restructuring care and treatment services. BMC Public Health. 2013; 13:86. Epub 2013/01/31. https://doi.org/10.1186/1471-2458-13-86 PMID: 23360397; PubMed Central PMCID: PMC3577494.

15. Saleem S, Bobak M. Women's autonomy, education and contraception use in Pakistan: a national study. Reprod Health. 2005; 2:8. Epub 2005/10/26. https://doi.org/10.1186/1742-4755-2-8 PMID: 16242030; PubMed Central PMCID: PMC1277848.

16. Kakaire O, Osinde MO, Kaye DK. Factors that predict fertility desires for people living with HIV infection at a support and treatment centre in Kabale, Uganda. Reprod Health. 2010; 7:27. Epub 2010/10/13. https://doi.org/10.1186/1742-4755-7-27 PMID: 20937095; PubMed Central PMCID: PMC2964526.

17. Quinn TC, Wawer MJ, Sewankambo N, Serwadda D, Li C, Wabwire-Mangen F, et al. Viral load and heterosexual transmission of human immunodeficiency virus type 1. 2000; 342(13):921-9. https://doi.org/ 10.1056/NEJM200003303421303 PMID: 10738050

18. Makwe CC, Giwa-Osagie OFJAjorh. Sexual and reproductive health in HIV serodiscordant couples. 2013; 17(4):99-106. PMID: 24689321

19. Agboghoroma CO, Giwa-Osagie OFJAjorh. Management of Infertility in HIV infected couples: A Review. 2012; 16(4):13-20. PMID: 23444539

20. Bujan L, Hollander L, Coudert M, Gilling-Smith C, Vucetich A, Guibert J, et al. Safety and efficacy of sperm washing in HIV-1-serodiscordant couples where the male is infected: results from the European CREAThE network. 2007; 21(14):1909-14. https://doi.org/10.1097/QAD.0b013e3282703879 PMID: 17721098

21. Aska ML, Chompikul J, Keiwkarnka BJWJoA. Determinants of fertility desires among HIV positive women living in the Western Highlands Province of Papua New Guinea. 2011; 1(04):198.

22. McClellana MK, Patel R, Kadzirangec G, Chipatod T, Katzenstein DJAJoRH. Fertility desires and condom use among HIV-positive women at an antiretroviral roll-out program in Zimbabwe. 2010; 14(2):2735. PMID: 21243916

23. Myer L, Morroni C, Rebe KJApc, STDs. Prevalence and determinants of fertility intentions of HIVinfected women and men receiving antiretroviral therapy in South Africa. 2007; 21(4):278-85. https:// doi.org/10.1089/apc.2006.0108 PMID: 17461723

24. Berhan Y, Berhan AJBPH. Meta-analyses of fertility desires of people living with HIV. 2013; 13(1):409. https://doi.org/10.1186/1471-2458-13-409 PMID: 23627965

25. Moher D, Liberati A, Tetzlaff J, Altman DGJAoim. Preferred reporting items for systematic reviews and meta-analyses: the PRISMA statement. 2009; 151(4):264-9. https://doi.org/10.7326/0003-4819-151-4200908180-00135 PMID: 19622511

26. Rostom A, Dubé C, Cranney A, Saloojee N, Sy R, Garritty C, et al. Celiac Disease. Rockville (MD): Agency for Healthcare Research and Quality (US); 2004 Sep.(Evidence Reports/Technology Assessments, No. 104.) Appendix D. Quality Assessment Forms. 2014.

27. Abbawa F, Awoke W, Alemu Y. Fertility desire and associated factors among clients on highly active antiretroviral treatment at finoteselam hospital Northwest Ethiopia: a cross sectional study. Reprod Health. 2015; 12:69. Epub 2015/08/12. https://doi.org/10.1186/s12978-015-0063-2 PMID: 26260021; PubMed Central PMCID: PMC4531807.

28. Adilo TM, Wordofa HM. Prevalence of fertility desire and its associated factors among 15- to 49-yearold people living with HIV/AIDS in Addis Ababa, Ethiopia: a cross-sectional study design. HIV AIDS (Auckl). 2017; 9:167-76. Epub 2017/09/19. https://doi.org/10.2147/HIV.S133766 PMID: 28919821; PubMed Central PMCID: PMC5587090.

29. Adler DH, Abar B, Bennie T, Sadeghi R, Bekker LG. Childbearing intentions among sexually active HIVinfected and HIV-uninfected female adolescents in South Africa. J AIDS HIV Res. 2017; 9(7):159-63. Epub 2017/12/08. https://doi.org/10.5897/JAHR2017.0432 PMID: 29214096; PubMed Central PMCID: PMC5714321.

30. Alemayehu B, Aregay AJJoA, Research H. Desire to procreate among people living with HIV/AIDS: Determinants in Ethiopia: a cross-sectional study. 2012; 4(5):128-35.

31. Asfaw HM, Gashe FE. Fertility intentions among HIV positive women aged 18-49 years in Addis Ababa Ethiopia: a cross sectional study. Reprod Health. 2014; 11:36. Epub 2014/06/03. https://doi.org/10. 1186/1742-4755-11-36 PMID: 24885318; PubMed Central PMCID: PMC4038077. 
32. Cohn SE, Haddad LB, Sheth AN, Hayford C, Chmiel JS, Janulis PF, et al., editors. Parenting Desires Among Individuals Living With Human Immunodeficiency Virus in the United States. Open forum infectious diseases; 2018: Oxford University Press US.

33. Cooper D, Moodley J, Zweigenthal V, Bekker LG, Shah I, Myer L. Fertility intentions and reproductive health care needs of people living with HIV in Cape Town, South Africa: implications for integrating reproductive health and HIV care services. AIDS Behav. 2009; 13 Suppl 1:38-46. Epub 2009/04/04 https://doi.org/10.1007/s10461-009-9550-1 PMID: 19343492.

34. De Souza MR, Do Amaral WN, Guimarães RA, Rezza G, Brunini SMJPo. Reproductive desire among women living with HIV/AIDS in Central Brazil: Prevalence and associated factors. 2017; 12(10): e0186267. https://doi.org/10.1371/journal.pone.0186267 PMID: 29053712

35. Demissie DB, Tebeje B, Tesfaye T. Fertility desire and associated factors among people living with HIV attending antiretroviral therapy clinic in Ethiopia. BMC Pregnancy Childbirth. 2014; 14:382. Epub 2014/ 11/21. https://doi.org/10.1186/s12884-014-0382-2 PMID: 25410125; PubMed Central PMCID: PMC4243275.

36. Erhabor O, Akani Cl, Eyindah CE. Reproductive health options among HIV-infected persons in the lowincome Niger Delta of Nigeria. HIV AIDS (Auckl). 2012; 4:29-35. Epub 2012/02/24. https://doi.org/10. 2147/HIV.S19413 PMID: 22359465; PubMed Central PMCID: PMC3284261.

37. Haddad LB, Machen LK, Cordes S, Huylebroeck B, Delaney A, Ofotokun I, et al. Future desire for children among women living with HIV in Atlanta, Georgia. AIDS Care. 2016; 28(4):455-9. Epub 2015/12/ 26. https://doi.org/10.1080/09540121.2015.1114996 PMID: 26702869.

38. Heard I, Sitta R, Lert F. Reproductive choice in men and women living with HIV: evidence from a large representative sample of outpatients attending French hospitals (ANRS-EN12-VESPA Study). Aids. 2007; 21 Suppl 1:S77-82. Epub 2006/12/13. https://doi.org/10.1097/01.aids.0000255089.44297.6f PMID: 17159592.

39. Hernando V, Alejos B, Alvarez D, Montero M, Perez-Elias MJ, Blanco JR, et al. Reproductive desire in women with HIV infection in Spain, associated factors and motivations: a mixed-method study. BMC Pregnancy Childbirth. 2014; 14:194. Epub 2014/06/07. https://doi.org/10.1186/1471-2393-14-194 PMID: 24902487; PubMed Central PMCID: PMC4063425.

40. Iliyasu Z, Abubakar IS, Kabir M, Babashani M, Shuaib F, Aliyu MH. Correlates of fertility intentions among HIV/AIDS patients in northern Nigeria. Afr J Reprod Health. 2009; 13(3):71-83. Epub 2010/08/ 10. PMID: 20690263.

41. Jose H, Madi D, Chowta N, Ramapuram J, Bhaskaran U, Achappa B, et al. Fertility Desires and Intentions among People Living with HIV/AIDS (PLWHA) in Southern India. J Clin Diagn Res. 2016; 10(6): Oc19-22. Epub 2016/08/10. https://doi.org/10.7860/JCDR/2016/20282.7968 PMID: 27504328; PubMed Central PMCID: PMC4963688.

42. Kaida A, Laher F, Strathdee SA, Janssen PA, Money D, Hogg RS, et al. Childbearing intentions of HIVpositive women of reproductive age in Soweto, South Africa: the influence of expanding access to HAART in an HIV hyperendemic setting. Am J Public Health. 2011; 101(2):350-8. Epub 2010/04/21. https://doi.org/10.2105/AJPH.2009.177469 PMID: 20403884; PubMed Central PMCID: PMC3020203.

43. Kawale P, Mindry D, Stramotas S, Chilikoh P, Phoya A, Henry K, et al. Factors associated with desire for children among HIV-infected women and men: a quantitative and qualitative analysis from Malawi and implications for the delivery of safer conception counseling. AIDS Care. 2014; 26(6):769-76. Epub 2013/11/07. https://doi.org/10.1080/09540121.2013.855294 PMID: 24191735; PubMed Central PMCID: PMC3943633.

44. Kipp W, Heys J, Jhangri GS, Alibhai A, Rubaale T. Impact of antiretroviral therapy on fertility desires among HIV-infected persons in rural Uganda. Reprod Health. 2011; 8:27. Epub 2011/10/07. https://doi. org/10.1186/1742-4755-8-27 PMID: 21975089; PubMed Central PMCID: PMC3214790.

45. Krashin JW, Haddad LB, Tweya H, Chiwoko J, Ng'ambi W, Samala B, et al. Factors associated with desired fertility among HIV-positive women and men attending two urban clinics in Lilongwe, Malawi. PLoS One. 2018; 13(6):e0198798. Epub 2018/06/14. https://doi.org/10.1371/journal.pone.0198798 PMID: 29897961; PubMed Central PMCID: PMC5999219.

46. Laar AK, Taylor AE, Akasoe BA. HIV-seropositivity is not important in childbearing decision-making among HIV-positive Ghanaian women receiving antiretroviral therapy. AIDS Care. 2015; 27(7):870-5. Epub 2015/02/05. https://doi.org/10.1080/09540121.2015.1007115 PMID: 25650646.

47. Litwin LE, Makumbi FE, Gray R, Wawer M, Kigozi G, Kagaayi J, et al. Impact of Availability and Use of ART/PMTCT Services on Fertility Desires of Previously Pregnant Women in Rakai, Uganda: A Retrospective Cohort Study. J Acquir Immune Defic Syndr. 2015; 69(3):377-84. Epub 2015/04/04. https:// doi.org/10.1097/QAI.0000000000000612 PMID: 25835605; PubMed Central PMCID: PMC4506706.

48. Maier M, Andia I, Emenyonu N, Guzman D, Kaida A, Pepper L, et al. Antiretroviral therapy is associated with increased fertility desire, but not pregnancy or live birth, among HIV+ women in an early HIV 
treatment program in rural Uganda. AIDS Behav. 2009; 13 Suppl 1:28-37. Epub 2008/04/05. https:// doi.org/10.1007/s10461-008-9371-7 PMID: 18389364; PubMed Central PMCID: PMC3606959.

49. Mayhew SH, Colombini M, Kimani JK, Tomlin K, Warren CE, Mutemwa R. Fertility intentions and contraceptive practices among clinic-users living with HIV in Kenya: a mixed methods study. BMC Public Health. 2017; 17(1):626. Epub 2017/07/07. https://doi.org/10.1186/s12889-017-4514-2 PMID: 28679389; PubMed Central PMCID: PMC5498886.

50. Mekonnen B, Minyihun A. Fertility Desire And Associated Factors Among HIV Positive Women Attending ART Clinics In Amhara Region Referral Hospitals In Northwest Ethiopia, 2017. Hiv Aids-Research and Palliative Care. 2019; 11:247-54. https://doi.org/10.2147/hiv.S221941 WOS:000490603300001. PMID: 31802952

51. Mekonnen $\mathrm{H}$, Enquselassie F. Effect of antiretroviral therapy on changes in the fertility intentions of human immunodeficiency virus-positive women in Addis Ababa, Ethiopia: a prospective follow-up study. Epidemiol Health. 2017; 39:e2017028. Epub 2017/07/22. https://doi.org/10.4178/epih.e2017028 PMID: 28728348; PubMed Central PMCID: PMC5668666.

52. Melaku YA, Zeleke EG, Kinsman J, Abraha AK. Fertility desire among HIV-positive women in Tigray region, Ethiopia: implications for the provision of reproductive health and prevention of mother-to-child HIV transmission services. BMC Womens Health. 2014; 14:137. Epub 2014/11/20. https://doi.org/10. 1186/s12905-014-0137-2 PMID: 25407330; PubMed Central PMCID: PMC4240867.

53. Moyo W, Mbizvo MT. Desire for a future pregnancy among women in Zimbabwe in relation to their selfperceived risk of HIV infection, child mortality, and spontaneous abortion. AIDS Behav. 2004; 8(1):915. Epub 2004/05/18. https://doi.org/10.1023/b:aibe.0000017521.26426.9d PMID: 15146129.

54. Myer L, Morroni C, Rebe K. Prevalence and determinants of fertility intentions of HIV-infected women and men receiving antiretroviral therapy in South Africa. AIDS Patient Care STDS. 2007; 21(4):278-85. Epub 2007/04/28. https://doi.org/10.1089/apc.2006.0108 PMID: 17461723.

55. Nakayiwa S, Abang B, Packel L, Lifshay J, Purcell DW, King R, et al. Desire for children and pregnancy risk behavior among HIV-infected men and women in Uganda. AIDS Behav. 2006; 10(4 Suppl):S95104. Epub 2006/05/23. https://doi.org/10.1007/s10461-006-9126-2 PMID: 16715343.

56. Nedjat S, Moazen B, Rezaei F, Hajizadeh S, Majdzadeh R, Setayesh HR, et al. Sexual and reproductive health needs of HIV-positive people in Tehran, Iran: a mixed-method descriptive study. Int J Health Policy Manag. 2015; 4(9):591-8. Epub 2015/09/05. https://doi.org/10.15171/ijhpm.2015.68 PMID: 26340488; PubMed Central PMCID: PMC4556575.

57. Nobrega AA, Oliveira FA, Galvao MT, Mota RS, Barbosa RM, Dourado I, et al. Desire for a child among women living with HIV/AIDS in northeast Brazil. AIDS Patient Care STDS. 2007; 21(4):261-7. Epub 2007/04/28. https://doi.org/10.1089/apc.2006.0116 PMID: 17461721.

58. Okome-Nkoumou M, Guiyedi V, Dzeing-Ella A, Komba-Boussaga $Y$, Efire-Emagha N, Menguet-Abessolo MP, et al. Socio-Demographic and Behavioral Factors Associated with the Desire to Procreate Among Patients Living with HIV in Gabon. Open AIDS J. 2015; 9:1-8. Epub 2015/02/04. https://doi.org/ 10.2174/1874613601509010001 PMID: 25646139; PubMed Central PMCID: PMC4311383.

59. Oladapo OT, Daniel OJ, Odusoga OL, Ayoola-Sotubo O. Fertility desires and intentions of HIV-positive patients at a suburban specialist center. J Natl Med Assoc. 2005; 97(12):1672-81. Epub 2006/01/07. PMID: 16396059; PubMed Central PMCID: PMC2640756.

60. Paiva V, Santos N, Franca-Junior I, Filipe E, Ayres JR, Segurado A. Desire to have children: gender and reproductive rights of men and women living with HIV: a challenge to health care in Brazil. AIDS Patient Care STDS. 2007; 21(4):268-77. Epub 2007/04/28. https://doi.org/10.1089/apc.2006.0129 PMID: 17461722.

61. Pottinger AM, Carroll K. Reproductive needs of PLHIV in Jamaica: relationship between fertility desire, motives and depression. J Reprod Infant Psychol. 2019:1-11. Epub 2019/04/04. https://doi.org/10. 1080/02646838.2019.1599334 PMID: 30939927.

62. Rhodes CM, Cu-Uvin S, Rana Al. Pregnancy Desire, Partner Serodiscordance, and Partner HIV Disclosure among Reproductive Age HIV-Infected Women in an Urban Clinic. Infect Dis Obstet Gynecol. 2016; 2016:8048457. Epub 2016/06/18. https://doi.org/10.1155/2016/8048457 PMID: 27313441; PubMed Central PMCID: PMC4899585.

63. Shiferaw T, Kiros G, Birhanu Z, Gebreyesus H, Berhe T, Teweldemedhin M. Fertility desire and associated factors among women on the reproductive age group of Antiretroviral treatment users in Jimma Town, South West Ethiopia. BMC Res Notes. 2019; 12(1):158. Epub 2019/03/22. https://doi.org/10. 1186/s13104-019-4190-7 PMID: 30894211; PubMed Central PMCID: PMC6425594.

64. Tamene W, Fantahun M. Fertility desire and family-planning demand among HIV-positive women and men undergoing antiretroviral treatment in Addis Ababa, Ethiopia. Afr J AIDS Res. 2007; 6(3):223-7. Epub 2007/11/01. https://doi.org/10.2989/16085900709490418 PMID: 25866168. 
65. Tesfaye L, Admassu M, Getachew A, Sharma HRJVC, Studies Y. Fertility desires and family planning demand among HIV-positive clients in follow-up care at antiretroviral treatment unit in Gondar university hospital, Ethiopia. 2012; 7(1):20-35.

66. Wagner G, Linnemayr S, Kityo C, Mugyenyi P. Factors associated with intention to conceive and its communication to providers among HIV clients in Uganda. Matern Child Health J. 2012; 16(2):510-8. Epub 2011/03/02. https://doi.org/10.1007/s10995-011-0761-5 PMID: 21359828.

67. Wagner GJ, Wanyenze R. Fertility Desires and Intentions and the Relationship to Consistent Condom Use and Provider Communication Regarding Childbearing Among HIV Clients in Uganda. ISRN Infect Dis. 2013;2013. Epub 2013/01/01. https://doi.org/10.5402/2013/478192 PMID: 25379322; PubMed Central PMCID: PMC4219363.

68. Wekesa E, Coast E. Fertility desires among men and women living with HIV/AIDS in Nairobi slums: a mixed methods study. PLoS One. 2014; 9(8):e106292. Epub 2014/08/30. https://doi.org/10.1371/ journal.pone.0106292 PMID: 25171593; PubMed Central PMCID: PMC4149552.

69. Thomson KA, Dhanireddy S, Andrasik M, Hancuch K, Legg L, Keane-Candib J, et al. Fertility desires and preferences for safer conception strategies among people receiving care for HIV at a publiclyfunded clinic in Seattle, WA. AIDS Care. 2018; 30(1):121-9. Epub 2017/10/27. https://doi.org/10.1080/ 09540121.2017.1390541 PMID: 29067843; PubMed Central PMCID: PMC6117831.

70. Pokharel R, Bhattarai G, Shrestha N, Onta S. Knowledge and utilization of family planning methods among people living with HIV in Kathmandu, Nepal. BMC Health Serv Res. 2018; 18(1):836. Epub 2018/11/08. https://doi.org/10.1186/s12913-018-3643-3 PMID: 30400972; PubMed Central PMCID: PMC6220510.

71. Laryea DO, Amoako YA, Spangenberg K, Frimpong E, Kyei-Ansong J. Contraceptive use and unmet need for family planning among HIV positive women on antiretroviral therapy in Kumasi, Ghana. BMC Womens Health. 2014; 14:126. Epub 2014/10/13. https://doi.org/10.1186/1472-6874-14-126 PMID: 25306546 ; PubMed Central PMCID: PMC4286913.

72. Gyimah AA, Nakua EK, Owusu-Dabo E, Otupiri E. Fertility Preferences of Women Living with HIV in the Kumasi Metropolis, Ghana. Afr J Reprod Health. 2015; 19(2):125-33. Epub 2015/10/29. PMID: 26506665.

73. Finocchario-Kessler S, Sweat MD, Dariotis JK, Trent ME, Kerrigan DL, Keller JM, et al. Understanding high fertility desires and intentions among a sample of urban women living with HIV in the United States. AIDS Behav. 2010; 14(5):1106-14. Epub 2009/11/13. https://doi.org/10.1007/s10461-009-9637-8 PMID: 19908135.

74. Finocchario Kessler S, Bastos FI, Malta M, Bertoni N, Hanif H, Kerrigan D. HIV+ men need reproductive counseling too: Assessing childbearing goals and provider communication among HIV+ male patients in Rio de Janeiro, Brazil. AIDS Patient Care STDS. 2014; 28(5):254-9. Epub 2014/05/07. https://doi. org/10.1089/apc.2013.0354 PMID: 24796758.

75. World Bank. List of economies.[Internet]. 2020 [cited 2020 Sep 25]. Available from: databank. worldbank.org/data/download/site-content/CLASS.xls.

76. Homsy J, Bunnell R, Moore D, King R, Malamba S, Nakityo R, et al. Reproductive intentions and outcomes among women on antiretroviral therapy in rural Uganda: a prospective cohort study. 2009; 4(1): e4149. https://doi.org/10.1371/journal.pone.0004149 PMID: 19129911

77. Negash S, Yusuf L, Tefera MJEmj. Fertility desires predictors among people living with HIV/AIDS at art care centers of two teaching hospitals in Addis Ababa. 2013; 51(1):1-11. PMID: 23930486

78. Cooper D, Harries J, Myer L, Orner P, Bracken H, Zweigenthal V. "Life is still going on": reproductive intentions among HIV-positive women and men in South Africa. Social science \& medicine (1982). 2007; 65(2):274-83. Epub 2007/04/25. https://doi.org/10.1016/j.socscimed.2007.03.019 PMID: 17451852.

79. Doyal L, Anderson J. 'My fear is to fall in love again. .. ' how HIV-positive African women survive in London. Social science \& medicine (1982). 2005; 60(8):1729-38. Epub 2005/02/03. https://doi.org/10. 1016/j.socscimed.2004.08.041 PMID: 15686805.

80. Paiva V, Filipe EV, Santos N, Lima TN, Segurado A. The right to love: the desire for parenthood among men living with HIV. Reproductive health matters. 2003; 11(22):91-100. Epub 2004/01/08. https://doi. org/10.1016/s0968-8080(03)02293-6 PMID: 14708400.

81. Awiti Ujiji O, Ekström AM, llako F, Indalo D, Rubenson B. "I will not let my HIV status stand in the way." Decisions on motherhood among women on ART in a slum in Kenya- a qualitative study. BMC Womens Health. 2010; 10:13. Epub 2010/04/29. https://doi.org/10.1186/1472-6874-10-13 PMID: 20423528; PubMed Central PMCID: PMC2873237.

82. Ngure K, Baeten JM, Mugo N, Curran K, Vusha S, Heffron R, et al. My intention was a child but I was very afraid: fertility intentions and HIV risk perceptions among HIV-serodiscordant couples experiencing 
pregnancy in Kenya. AIDS Care. 2014; 26(10):1283-7. Epub 2014/05/02. https://doi.org/10.1080/ 09540121.2014.911808 PMID: 24779445; PubMed Central PMCID: PMC4087054.

83. Mokwena K, Bogale YR. Fertility intention and use of contraception among women living with the human immunodeficiency virus in Oromia Region, Ethiopia. South African Family Practice. 2017; 59 (1):46-+. https://doi.org/10.1080/20786190.2016.1254931 WOS:000397055400016.

84. Schwartz SR, Rees H, Mehta S, Venter WDF, Taha TE, Black VJPo. High incidence of unplanned pregnancy after antiretroviral therapy initiation: findings from a prospective cohort study in South Africa. 2012; 7(4):e36039. https://doi.org/10.1371/journal.pone.0036039 PMID: 22558319

85. Davey DLJ, Wall KM, Kilembe W, Khu NH, Brill I, Vwalika B, et al. Difficult decisions: Evaluating individual and couple-level fertility intentions and HIV acquisition among HIV serodiscordant couples in Zambia. 2018; 13(1):e0189869. https://doi.org/10.1371/journal.pone.0189869 PMID: 29364895

86. Kodzi IA, Johnson DR, Casterline JBJDR. Examining the predictive value of fertility preferences among Ghanaian women. 2010; 22:965. https://doi.org/10.4054/DemRes.2010.22.30 PMID: 23970826

87. Guthrie BL, de Bruyn G, Farquhar CJCHr. HIV-1-discordant couples in sub-Saharan Africa: explanations and implications for high rates of discordancy. 2007; 5(4):416-29. https://doi.org/10.2174/ 157016207781023992 PMID: 17627505

88. Dunkle KL, Stephenson R, Karita E, Chomba E, Kayitenkore K, Vwalika C, et al. New heterosexually transmitted HIV infections in married or cohabiting couples in urban Zambia and Rwanda: an analysis of survey and clinical data. 2008; 371(9631):2183-91. https://doi.org/10.1016/S0140-6736(08)60953-8 PMID: 18586173

89. Ngure K, Mugo N, Celum C, Baeten JM, Morris M, Olungah O, et al. A qualitative study of barriers to consistent condom use among HIV-1 serodiscordant couples in Kenya. 2012; 24(4):509-16. https://doi. org/10.1080/09540121.2011.613911 PMID: 22085306 